\title{
Evolution of the Minimax Inequality of Ky Fan
}

\author{
Sehie Park ${ }^{1,2}$ \\ ${ }^{1}$ The National Academy of Sciences, ROK, Seoul 137-044, Republic of Korea \\ ${ }^{2}$ Department of Mathematical Sciences, Seoul National University, Seoul 151-747, Republic of Korea
}

Correspondence should be addressed to Sehie Park; shpark@math.snu.ac.kr

Received 14 November 2012; Accepted 27 February 2013

Academic Editor: Ram U. Verma

Copyright (c) 2013 Sehie Park. This is an open access article distributed under the Creative Commons Attribution License, which permits unrestricted use, distribution, and reproduction in any medium, provided the original work is properly cited.

There are quite a few generalizations or applications of the 1984 minimax inequality of Ky Fan compared with his original 1972 minimax inequality. In a certain sense, the relationship between the 1984 inequality and several hundreds of known generalizations of the original 1972 inequality has not been recognized for a long period. Hence, it would be necessary to seek such relationship. In this paper, we give several generalizations of the 1984 inequality and some known applications in order to clarify the close relationship among them. Some new types of minimax inequalities are added.

\section{Introduction}

The KKM theory is originated from the Knaster-KuratowskiMazurkiewicz (KKM for short) theorem of 1929 [1]. Since then, it has been found a large number of results which are equivalent to the KKM theorem; see $[2,3]$. Typical examples of the most remarkable and useful equivalent formulations are Ky Fan's KKM lemma of 1961 [4] and his minimax inequality of 1972 [5]. The inequality and its various generalizations are very useful tools in various fields of mathematical sciences.

Since 1961, Ky Fan showed that the KKM theorem provides the foundation for many of the modern essential results in diverse areas of mathematical sciences. Actually, a milestone in the history of the KKM theory was erected by Fan in 1961 [4]. His 1961 KKM Lemma (or the Fan-KKM theorem) extended the KKM theorem to arbitrary topological vector spaces and had been applied to various problems in his subsequent papers [5-10].

Recall that, at the beginning, the basic theorems in the KKM theory and their applications were established for convex subsets of topological vector spaces mainly by Fan in 1961-1984 [4-10]. A number of intersection theorems and their applications to various equilibrium problems followed. In our previous review [11], we recalled Fan's contributions to the KKM theory based on his celebrated 1961 KKM lemma, and introduced relatively recent applications of the lemma due to other authors in the twenty-first century.

Then, the KKM theory was extended to convex spaces by Lassonde in 1983 [12] and to $c$-spaces (or H-spaces) by Horvath in 1983-1993 [13-16] and others. Since 1993, the theory has been extended to generalized convex (G-convex) spaces in a sequence of papers of the present author and others; see [2]. Since 2006, the main theme of the theory has become abstract convex spaces in the sense of Park [17-30]. The basic theorems in the theory have numerous applications to various equilibrium problems in nonlinear analysis and other fields. In our previous review [30], we recalled our versions of general KKM type theorems for abstract convex spaces and introduced relatively recent applications of various generalized KKM type theorems due to other authors in the twenty-first century.

While we were studying on [11,30], we noticed that there are quite a few generalizations and applications of the 1984 minimax inequality of Fan compared with his 1972 inequality. In a certain sense, the 1984 inequality is not connected with several hundreds of known generalizations of the original minimax inequality. Hence, it would be necessary to seek such relationship. In this paper, we introduce several generalizations of two minimax inequalities of Fan and some known direct applications in order to clarify the close relationship among them. 
This paper is organized as follows. In Section 2, we introduce Fan's 1961 KKM lemma, 1972 minimax inequality, and 1984 minimax inequality with some applications of them. Section 3 deals with the evolution of the 1984 inequality. Actually, we give its generalizations to convex spaces of Lassonde, convex spaces with the coercivity due to Chang, $\mathrm{H}_{-}$ spaces originated by Horvath, and G-convex spaces and $\phi_{A^{-}}$ spaces of Park. In Section 4, we introduce recently obtained basic concepts or results of the KKM theory such as the abstract convex spaces, generalized KKM theorems and general minimax inequalities. We show that one of our general minimax inequalities subsumes previously obtained inequalities on various types of KKM spaces in Section 3. Section 5 deals with other generalizations of the Fan minimax inequality and vector minimax inequality. Finally, we add some related historical remarks in Section 6.

\section{The Origin and Fan's Applications}

The KKM theorem of Knaster, Kuratowski, and Mazurkiewicz of 1929 [1] was extended by Fan in 1961 [4] as follows.

The 1961 KKM Lemma of Fan. Let $X$ be an arbitrary set in a Hausdorff topological vector space $Y$. To each $x \in X$, let a closed set $F(x)$ in $Y$ be given such that the following two conditions are satisfied:

(i) convex hull of any finite subset $\left\{x_{1}, \ldots, x_{n}\right\}$ of $X$ is contained in $\bigcup_{i=1}^{n} F\left(x_{i}\right)$,

(ii) $F(x)$ is compact for at least one $x \in X$.

Then $\bigcap_{x \in X} F(x) \neq \emptyset$.

This is usually known as the Fan-KKM lemma, the Fan$K K M$ theorem, or the KKMF theorem. Fan and his followers applied his KKM lemma to various problems in many fields in mathematics; see [2, 29].

Five decades after the birth of the lemma, the above original form is still adopted by many authors in each year. But, it was found quite a long time ago by Lassonde [12] that the Hausdorffness is redundant. Moreover, note that $Y$ can be any convex subset of a topological vector space.

Recall that an extended real-valued function $f: X \rightarrow \overline{\mathbb{R}}$, where $X$ is a topological space, is lower semicontinuous (1.s.c.) if $\{x \in X: f(x)>r\}$ is open for each $r \in \overline{\mathbb{R}}$.

For a convex set $X$, a function $f: X \rightarrow \overline{\mathbb{R}}$ is said to be quasiconcave if $\{x \in X: f(x)>r\}$ is convex for each $r \in \overline{\mathbb{R}}$.

Similarly, the upper semicontinuity (u.s.c.) and the quasiconvexity can be defined.

One of the most remarkable equivalent formulations of the KKM theorem is the minimax inequality established by Fan from his KKM lemma. The following is the original form given by Fan in 1972 [5].

The Fan Minimax Inequality. Let $X$ be a compact convex set in a Hausdorff topological vector space. Let $f$ be a real-valued function defined on $X \times X$ such that

(a) for each fixed $x \in X, f(x, y)$ is a lower semicontinuous function of $y$ on $X$; (b) for each fixed $y \in X, f(x, y)$ is a quasiconcave function of $x$ on $X$.

Then, the minimax inequality

$$
\min _{y \in X} \sup _{x \in X} f(x, y) \leq \sup _{x \in X} f(x, x)
$$

holds.

In [10], Fan applied his inequality to the following:

(1) a variational inequality (extending Hartman-Stampacchia (1966) and Browder (1967));

(2) a geometric formulation of the inequality (equivalent to the Fan-Browder fixed point theorem (1968));

(3) separation properties of u.d.c. multimaps, coincidence and fixed point theorems;

(4) properties of sets with convex sections (from which the Sion minimax theorem (1958), the equilibrium theorem of Nash (1951), and a variant of a theorem of Debrunner and Flor (1964) on extension of monotone sets easily follow);

(5) a fundamental existence theorem in potential theory.

The inequality became a crucial tool in proving many existence problems in various fields of mathematical sciences, for example, nonlinear analysis, especially in fixed point theory, variational inequality problems, various equilibrium theory, mathematical programming, partial differential equations, game theory, impulsive control, and mathematical economics; see $[21,26,31]$ and the references therein.

Fan [10] noted that the following 1984 generalized minimax inequality extending Allen's variational inequality of 1977 [32] follows from his 1984 KKM theorem.

Theorem 1 (see [10]). Let $X$ be a nonempty convex set in a Hausdorff topological vector space. Let $f$ be a real-valued function defined on $X \times X$ such that

(a) for each fixed $x \in X, f(x, y)$ is a lower semicontinuous function of $y$ on $X$;

(b) for each fixed $y \in X, f(x, y)$ is a quasi-concave function of $x$ on $X$;

(c) $f(x, x) \leq 0$ for all $x \in X$;

(d) $X$ has a nonempty compact convex subset $X_{0}$ such that the set $\left\{y \in X: f(x, y) \leq 0\right.$ for all $\left.x \in X_{0}\right\}$ is compact.

Then, there exists a point $\hat{y} \in X$ such that $f(x, \hat{y}) \leq 0$ for all $x \in X$.

The following proof was given by Fan [10].

Theorem $1 \Rightarrow$ The Fan Minimax Inequality. Using condition (a), we see that in the case compact $X$, condition (d) is fulfilled by taking $X_{0}$ as any nonempty closed convex subset of $X$. Thus, Theorem 1 reduces to the minimax inequality.

In [10], Theorem 1 was applied to a best approximation theorem, coincidence and fixed point theorems, a matching 
theorem for two closed covers of a convex set, another proof of the Brouwer fixed point theorem, and a generalization of Shapley's KKM theorem.

Recall that the original Nash equilibrium theorem was proved by the Brouwer or the Kakutani fixed point theorem; see [33, 34]. Later, Fan [7] proved it by applying his result on sets with convex sections. Nowadays, it is known to be one of the most important applications of the Fan minimax inequality; see $[10,27]$. Note that, in a wide sense, the Brouwer theorem, the KKM theorem, the Kakutani theorem, the Nash theorem, Fan's theorem on sets with convex sections, the Fan inequality, the Fan-Browder fixed point theorem, and many others are mutually equivalent; see $[2,3]$.

\section{Evolution of the Minimax Inequality of Ky Fan}

The Fan minimax inequality has been followed by a large number of generalizations and applications in the KKM theory on convex subsets of topological vector spaces, Lassonde type convex spaces, Horvath type $\mathrm{H}$-spaces, generalized convex spaces due to Park, and other types of spaces. Furthermore, many authors generalized the lower semicontinuity and quasiconcavity in the inequality or replaced them by other requirements. Therefore, even for convex spaces, it is necessary to establish proper forms of the Fan minimax inequality which unify as many particular cases as possible.

Multimaps are also simply called maps. Let $\langle D\rangle$ denote the set of all nonempty finite subsets of a set $D$.

In this section, we show several major generalizations of the Fan minimax inequality in the chronological order.

3.1. Lassonde's Theorem. The concept of convex sets in a topological vector space was extended to convex spaces by Lassonde in 1983 [12], and further to $c$-spaces by Horvath in 1983-1993 [13-16]. A number of other authors also extended the concept of convexity for various purposes.

Definition 2. Let $X$ be a subset of a vector space and $D$ a nonempty subset of $X$. One calls $(X, D)$ a convex space if co $D \subset X$ and $X$ has a topology that induces the Euclidean topology on the convex hulls of any $N \in\langle D\rangle$ (see Park [35]). For a convex space $(X, D)$, a subset $C$ of $X$ is said to be $D$ convex if for each $A \in\langle D\rangle, A \subset C$ implies $\operatorname{co} A \subset C$.

If $X=D$ is convex, then $X=(X, X)$ becomes a convex space in the sense of Lassonde [12].

A nonempty subset $L$ of a convex space $X$ is called a $c$ compact set [12] if for each finite subset $S \subset X$ there is a compact convex set $L_{S} \subset X$ such that $L \cup S \subset L_{S}$.

Lassonde [12] presented a simple and unified treatment of a large variety of minimax and fixed point problems. He first noticed that the Hausdorffness in the 1961 Fan-KKM lemma is redundant. More specifically, he gave several KKM type theorems for convex spaces $(X, D)$ and proposed a systematic development of the method based on the KKM theorem; the principal topics treated by him may be listed as follows:

Fixed point theory for multimaps;

Minimax equalities;
Extensions of monotone sets;
Variational inequalities;
Special best approximation problems.

The following [12, Proposition I.1.4] extends the 1984 inequality of Fan.

Theorem 3 (see [12]). Let $X$ be a convex space and $\varphi: X \rightarrow$ $\mathbb{R} \cup\{+\infty\}$ a convex l.s.c. function satisfying the following:

(i) for each fixed $x \in X, y \mapsto f(x, y)$ is l.s.c. on (compact subsets of) $X$;

(ii) for each fixed $y \in X, x \mapsto f(x, y)$ is concave on $X$;

(iii) for all $x \in X, f(x, x) \leq 0$.

Furthermore, assume that the following "coercivity" condition holds:

(iv) there are a compact set $K \subset X$ and a c-compact set $L \subset X$ such that for each $y \in X \backslash K$, there is an $x \in L$ with $f(x, y)+\varphi(y)>\varphi(x)$.

Then, there exists $y_{0} \in X$ such that $f\left(x, y_{0}\right)+\varphi\left(y_{0}\right) \leq \varphi(x)$ for all $x \in X$.

Consider that the following extends Theorem 3.

Theorem $3^{\prime}$. In Theorem 3, assume that

(ii)' for each fixed $y \in X, x \mapsto f(x, y)$ is quasiconcave on $X$ instead of (ii).

Theorem $3^{\prime} \Rightarrow$ Theorem 1 . Note that (a)-(c) of Theorem 1 imply (i), (ii) ${ }^{\prime}$, and (iii) of Theorem $3^{\prime}$, respectively. Now, we show that (d) implies (iv). Let $K:=\{y \in X: f(x, y) \leq 0$ for all $\left.x \in X_{0}\right\}$. Then for all $y \in X \backslash K$, there exists $x \in X_{0}$ such that $f(x, y)>0$. Since $X_{0}$ is a compact convex subset of a Hausdorff topological vector space, it is a $c$-compact set. Then, condition (iv) holds with $L:=X_{0}$ and $\varphi \equiv 0$. Therefore, by Theorem $3^{\prime}$, there exists a point $\hat{y} \in X$ such that $f(x, \widehat{y}) \leq 0$ for all $x \in X$.

Remark 4. (1) As shown in the above proof, the Hausdorffness in the 1984 inequality is essential. However, it is redundant in the original 1972 minimax inequality.

(2) The compactly closed (resp., open) sets adopted by Lassonde and many followers can be replaced simply by closed (resp., open) sets; see [22, 36]. Similarly, "l.s.c. on

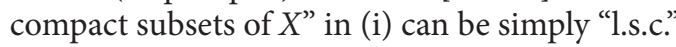

3.2. Chang Type Coercivity. In 1989, Chang [37] obtained a KKM theorem with a coercivity condition which eliminated the concept of $c$-compact sets. From a Fan-Browder type fixed point theorem equivalent to her KKM theorem, we noticed that the following holds [38].

Theorem 5 (see [38]). Let $X$ be a convex space, and $p, q: X \times$ $X \rightarrow \mathbb{R} \cup\{+\infty\}$ and $h: X \rightarrow \mathbb{R} \cup\{+\infty\}$ be functions satisfying the following: 
(i) $q(x, y) \leq p(x, y)$ for $(x, y) \in X \times X$ and $p(x, x) \leq 0$ for all $x \in X$;

(ii) for each $y \in X,\{x \in X: p(x, y)+h(y)>h(x)\}$ is convex or empty;

(iii) for each $x \in X,\{y \in X: q(x, y)+h(y)>h(x)\}$ is open;

(iv) there exist a nonempty compact subset $K$ of $X$ and, for each finite subset $N$ of $X$, a compact convex subset $L_{N}$ of $X$ containing $N$ such that $y \in L_{N} \backslash K$ implies $q(x, y)+h(y)>h(x)$ for some $x \in L_{N}$.

Then, there exists a point $y_{0} \in K$ such that

$$
q\left(x, y_{0}\right)+h\left(y_{0}\right) \leq h(x) \quad \forall x \in X
$$

Moreover, the set of all such solutions $y_{0}$ is a compact subset of K.

Actually, Theorem 5 is equivalent to the following.

Theorem $5^{\prime}$. The case of $h \equiv 0$ in Theorem 5 .

Theorem $5 \Rightarrow$ Theorem 3 . We apply Theorem 5 with $p(x, y)=$ $q(x, y)=f(x, y)$ and $h(x)=\varphi(x)$. Then we obtain (i)-(iv) in Theorem 5 from Theorem 3 as follows.

(i) Clearly holds since $f(x, x) \leq 0$ for all $x \in X$.

(ii) For each $y \in X$,

$$
\begin{aligned}
\{x & \in X: f(x, y)+\varphi(y)>\varphi(x)\} \\
& =\{x \in X: f(x, y)-\varphi(x)>-\varphi(y)\}
\end{aligned}
$$

is convex or empty since $x \mapsto f(x, y)$ is concave and $x \mapsto \varphi(x)$ is convex.

(iii) For each $x \in X,\{y \in X: f(x, y)+\varphi(y)>\varphi(x)\}$ is open since $y \mapsto f(x, y)$ is l.s.c. and $y \mapsto \varphi(y)$ is l.s.c.

(iv) There exist a nonempty compact subset $K$ of $X$ and, for each finite subset $N$ of $X$, a compact convex subset $L_{N}$ of $X$ containing $L \cup N$ (since $L$ is $c$-compact) such that $y \in L_{N} \backslash K \subset X \backslash K$ implies $f(x, y)+\varphi(y)>\varphi(x)$ for some $x \in L \subset L_{N}$.

Therefore, all of the requirements of Theorem 5 are satisfied. Therefore, the conclusion of Theorem 3 follows from Theorem 5 .

\subsection{For H-Spaces. In this subsection, we follow [39].}

Definition 6. A triple $(X, D ; \Gamma)$ is called an $\mathrm{H}$-space if $X$ is a topological space, $D$ is a nonempty subset of $X$, and $\Gamma=\left\{\Gamma_{A}\right\}$ is a family of contractible (or, more generally, $\omega$-connected) subsets of $X$ indexed by $A \in\langle D\rangle$ such that $\Gamma_{A} \subset \Gamma_{B}$ whenever $A \subset B \in\langle D\rangle$.

For an $\mathrm{H}$-space $(X, D ; \Gamma)$, a subset $C$ of $X$ is said to be $\mathrm{H}$ convex if for each $A \in\langle D\rangle, A \subset C$ implies $\Gamma_{A} \subset C$.

A multimap $F: D \multimap X$ is said to be $\mathrm{H}-\mathrm{KKM}$ if $\Gamma_{A} \subset F(A)$ for each $A \in\langle D\rangle$. A subset $L$ of $X$ is called an $\mathrm{H}$-subspace of
$(X, D ; \Gamma)$ if $L \cap D \neq \emptyset$, and for every $A \in\langle L \cap D\rangle, \Gamma_{A} \cap L$ is contractible.

If $D=X$, we denote $(X ; \Gamma)$ instead of $(X, X ; \Gamma)$, which is called a $c$-space by Horvath [13-16] or an $\mathrm{H}$-space by Bardaro and Ceppitelli [40]. Horvath noted that a torus, the Möbius band, or the Klein bottle can be regarded as $c$-spaces and are examples of compact $c$-spaces without having the fixed point property.

In the frame of $\mathrm{H}$-spaces, we obtained several generalized minimax inequalities. The following is one of them in [39, Theorem 7].

Theorem 7 (see [39]). Let $(X, D ; \Gamma)$ be an $H$-space, let $\phi: D \times$ $X \rightarrow \overline{\mathbb{R}}$ and $\psi: X \times X \rightarrow \overline{\mathbb{R}}$ be functions, and let $\gamma \in \overline{\mathbb{R}}$ such that

(1) $\phi(x, y) \leq \psi(x, y)$ for all $(x, y) \in D \times X$ and $\psi(x, x) \leq$ $\gamma$ for all $x \in X$,

(2) for each $x \in D,\{y \in X: \phi(x, y) \leq \gamma\}$ is (compactly) closed,

(3) for each $y \in X,\{x \in X: \psi(x, y)>\gamma\}$ is H-convex.

Suppose that there exists a nonempty compact subset $K$ of $X$ such that either

(i) there exists an $M \in\langle D\rangle$ such that

$$
\{y \in X: \phi(x, y) \leq \gamma \forall x \in M\} \subset K,
$$

(ii) or for each $N \in\langle D\rangle$, there exists a compact $H$-subspace $L_{N}$ of $X$ containing $N$ such that for each $y \in L_{N} \backslash K$ there exists an $x \in L_{N} \cap D$ satisfying $\phi(x, y)>\gamma$.

Then, there exists a $\hat{y} \in K$ such that

$$
\sup _{x \in D} \phi(x, \hat{y}) \leq \gamma .
$$

Theorem $7 \Rightarrow$ Theorem $5^{\prime}$. Any convex space is an $\mathrm{H}$-space.

Note that Theorem 7 extends all of Theorems 1-5, 3', and $5^{\prime}$ and that Theorem 7 with the coercivity condition (i) generalizes the original minimax inequality of Fan.

3.4. For G-Convex Spaces. Since 1996 the following became one of the main themes of the KKM theory $[41,42]$.

Definition 8. A generalized convex space or a G-convex space $(X, D ; \Gamma)$ consists of a topological space $X$, a nonempty set $D$, and a map $\Gamma:\langle D\rangle \multimap X$ such that for each $A \in\langle D\rangle$ with the cardinality $|A|=n+1$, there exists a continuous function $\phi_{A}: \Delta_{n} \rightarrow \Gamma(A)$ such that $J \in\langle A\rangle$ implies $\phi_{A}\left(\Delta_{J}\right) \subset \Gamma(J)$.

Here, $\Delta_{n}=\operatorname{co}\left\{e_{i}\right\}_{i=0}^{n}$ is the standard $n$-simplex, and $\Delta_{J}$ is the face of $\Delta_{n}$ corresponding to $J \in\langle A\rangle$; that is, if $A=$ $\left\{a_{0}, a_{1}, \ldots, a_{n}\right\}$ and $J=\left\{a_{i_{0}}, a_{i_{1}}, \ldots, a_{i_{k}}\right\} \subset A$, then $\Delta_{J}=$ $\operatorname{co}\left\{e_{i_{0}}, e_{i_{1}}, \ldots, e_{i_{k}}\right\}$. We may write $\Gamma_{A}=\Gamma(A)$ for each $A \in\langle D\rangle$ and $(X ; \Gamma)=(X, X ; \Gamma)$.

For a $\mathrm{G}$-convex space $(X, D ; \Gamma)$, a subset $C$ of $X$ is said to be G-convex with respect some $D^{\prime} \subset D$ if for each $N \in\left\langle D^{\prime}\right\rangle$, we have $\Gamma_{N} \subset C$.

A map $F: D \multimap X$ is called a KKM map if $\Gamma_{N} \subset F(N)$ for each $N \in\langle D\rangle$. 
There are lots of examples of G-convex spaces; see $[3,41]$ and the references therein. So, the KKM theory was extended to the study of KKM maps on G-convex spaces.

The following minimax inequality for G-convex spaces originates from $[41,42]$.

Theorem 9. Let $(X, D ; \Gamma)$ be a $G$-convex space, let $\phi: D \times X \rightarrow$ $\overline{\mathbb{R}}$ and $\psi: X \times X \rightarrow \overline{\mathbb{R}}$ be functions, and let $\gamma \in \overline{\mathbb{R}}$ such that

(1) $\psi(x, x) \leq \gamma$ for all $x \in X$,

(2) for each $z \in D,\{y \in X: \phi(z, y) \leq \gamma\}$ is closed,

(3) for each $y \in X, N \in\langle\{z \in D: \phi(x, y)>\gamma\}\rangle$ implies $\Gamma_{N} \subset\{x \in X: \psi(x, y)>\gamma\}$.

Suppose that there exists a nonempty compact subset $K$ of $X$ such that either

(i) there exists an $M \in\langle D\rangle$ such that

$$
\{y \in X: \phi(z, y) \leq \gamma \forall z \in M\} \subset K,
$$

(ii) or for each $N \in\langle D\rangle$, there exists a compact G-convex subset $L_{N}$ of $X$ relative to some $D^{\prime} \subset D$ such that $N \subset$ $D^{\prime}$, and for each $y \in L_{N} \backslash K$ there exists a $z \in D^{\prime}$ satisfying $\phi(z, y)>\gamma$.

Then, there exists $a \hat{y} \in K$ such that

$$
\sup _{z \in D} \phi(z, \hat{y}) \leq \gamma
$$

Theorem $9 \Rightarrow$ Theorem 7 . Any H-space is a G-convex space.

3.5. For $\phi_{A}$-Spaces. Since 2007, the following became one of the main themes of the KKM theory $[17,43,44]$.

Definition 10. A space having a family $\left\{\phi_{A}\right\}_{A \in\langle D\rangle}$ or simply a $\phi_{A}$-space

$$
\left(X, D ;\left\{\phi_{A}\right\}_{A \in\langle D\rangle}\right) \text { or simply }\left(X, D ; \phi_{A}\right)
$$

consists of a topological space $X$, a nonempty set $D$, and a family of continuous functions $\phi_{A}: \Delta_{n} \rightarrow X$ (i.e., singular $n$-simplexes) for $A \in\langle D\rangle$ with the cardinality $|A|=n+1$.

For a $\phi_{A}$-space $\left(X, D ; \phi_{A}\right)$, a subset $C$ of $X$ is said to be $\phi_{A}$-convex relative to some $D^{\prime} \subset D$ if for each $A \in\left\langle D^{\prime}\right\rangle$, we have $\phi_{A}\left(\Delta_{|A|-1}\right) \subset C$.

We define a KKM map $G: D \multimap X$ on a $\phi_{A}$-space $\left(X, D ; \phi_{A}\right)$ if, for each $N \in\langle D\rangle$ and $J \subset N$, we have

$$
\phi_{N}\left(\Delta_{|J|-1}\right) \subset G(J) \text {, }
$$

where $\Delta_{|J|-1}$ is the face of $\Delta_{|N|-1}$ corresponding to J; see Park [17].

There are lots of examples of $\phi_{A}$-spaces; see $[3,17,43,44]$ and the references therein. So, the KKM theory was extended to the study of KKM maps on $\phi_{A}$-spaces.

The following minimax inequality for $\phi_{A}$-spaces is new.
Theorem 11. Let $\left(X, D ; \phi_{A}\right)$ be a $\phi_{A}$-space, let $f: D \times X \rightarrow \overline{\mathbb{R}}$ and $g: X \times X \rightarrow \overline{\mathbb{R}}$ be functions, and let $\gamma \in \overline{\mathbb{R}}$ such that

(1) $g(x, x) \leq \gamma$ for all $x \in X$,

(2) for each $z \in D,\{y \in X: f(z, y) \leq \gamma\}$ is closed,

(3) for each $y \in X$ and $A \in\langle\{z \in D: f(z, y)>\gamma\}\rangle$, one has $\phi_{A}\left(\Delta_{|A|-1}\right) \subset\{x \in X: g(x, y)>\gamma\}$.

Suppose that there exists a nonempty compact subset $K$ of $X$ such that either

(i) there exists an $M \in\langle D\rangle$ such that

$$
\{y \in X: \phi(z, y) \leq \gamma \forall z \in M\} \subset K,
$$

(ii) or for each $N \in\langle D\rangle$, there exist a compact $\phi_{A}$-convex subset $L_{N}$ of $X$ relative to some $D^{\prime} \subset D$ such that $N \subset$ $D^{\prime}$ and for each $y \in L_{N} \backslash K$ there exists a $z \in D^{\prime}$ satisfying $f(z, y)>\gamma$.

Then, there exists a $\hat{y} \in K$ such that

$$
\sup _{z \in D} f(z, \widehat{y}) \leq \gamma .
$$

Theorem $11 \Rightarrow$ Theorem 9. Any G-convex space is a $\phi_{A}$-space such that $\phi_{A}\left(\Delta_{|A|-1}\right) \subset \Gamma_{A}$ for each $A \in\langle D\rangle$. Therefore, by putting $f:=\phi, g:=\psi$, and $\phi_{A}\left(\Delta_{|A|-1}\right):=\Gamma_{A}$, Theorem 11 reduces to Theorem 9 .

\section{Abstract Convex Spaces and General KKM Theorems}

Recall the following in $[3,18,19]$.

Definition 12. An abstract convex space $(E, D ; \Gamma)$ consists of a topological space $E$, a nonempty set $D$, and a multimap $\Gamma$ : $\langle D\rangle \multimap E$ with nonempty values $\Gamma_{A}:=\Gamma(A)$ for $A \in\langle D\rangle$.

For any $D^{\prime} \subset D$, the $\Gamma$-convex hull of $D^{\prime}$ is denoted and defined by

$$
\operatorname{co}_{\Gamma} D^{\prime}:=\bigcup\left\{\Gamma_{A}: A \in\left\langle D^{\prime}\right\rangle\right\} \subset E
$$

A subset $X$ of $E$ is called a $\Gamma$-convex subset of $(E, D ; \Gamma)$ relative to $D^{\prime}$ if for any $N \in\left\langle D^{\prime}\right\rangle$, we have $\Gamma_{N} \subset X$; that is, $\mathrm{co}_{\Gamma} D^{\prime} \subset X$.

In case $E=D$, let $(E ; \Gamma):=(E, E ; \Gamma)$.

Definition 13. Let $(E, D ; \Gamma)$ be an abstract convex space and $Z$ be a topological space. For a multimap $F: E \multimap Z$ with nonempty values, if a multimap $G: D \multimap Z$ satisfies

$$
F\left(\Gamma_{A}\right) \subset G(A):=\bigcup_{y \in A} G(y) \quad \forall A \in\langle D\rangle,
$$

then $G$ is called a KKM map with respect to $F$. A KKM map $G: D \multimap E$ is a KKM map with respect to the identity map $1_{E}$.

A multimap $F: E \multimap Z$ is called a $\mathfrak{\Re} \mathfrak{C}$-map (resp., a $\mathfrak{R D}$-map) if, for any closed-valued (resp., open-valued) KKM map $G: D \multimap Z$ with respect to $F$, the family $\{G(y)\}_{y \in D}$ has the finite intersection property. In this case, we denote $F \in \mathfrak{K} \mathfrak{C}(E, D, Z)$ (resp., $F \in \mathfrak{K D}(E, D, Z))$. 
Definition 14. The partial KKM principle for an abstract convex space $(E, D ; \Gamma)$ is the statement $1_{E} \in \mathfrak{I} \mathfrak{S}(E, D, E)$; that is, for any closed-valued KKM map $G: D \multimap E$, the family $\{G(y)\}_{y \in D}$ has the finite intersection property. The $K K M$ principle is the statement that the same property also holds for any open-valued KKM map.

An abstract convex space is called a (partial) KKM space if it satisfies the (partial) KKM principle, respectively.

In our recent works $[3,18,19]$, we studied the elements or foundations of the KKM theory on abstract convex spaces and noticed that many important results therein are related to the partial KKM principle.

Example 15. We give known examples of partial KKM spaces; see [3] and the references therein.

(1) Every $\phi_{A}$-space is a KKM space. More precisely, for a $\phi_{A}$-space $\left(X, D ; \phi_{A}\right)$, the corresponding abstract convex space $(X, D ; \Gamma)$ with $\Gamma_{A}:=\phi_{A}\left(\Delta_{n}\right)$ for $A \epsilon$ $\langle D\rangle,|A|=n+1$, is a KKM space. This KKM space may not be $G$-convex; see [25].

(2) A connected linearly ordered space $(X, \leq)$ can be made into a KKM space.

(3) The extended long line $L^{*}$ is a $\operatorname{KKM}$ space $\left(L^{*}, D ; \Gamma\right)$ with the ordinal space $D:=[0, \Omega]$. But $L^{*}$ is not a G-convex space.

(4) For a closed convex subset $X$ of a complete $\mathbb{R}$-tree $H$, and $\Gamma_{A}:=\operatorname{conv}_{H}(A)$ for each $A \in\langle X\rangle$, Kirk and Panyanak showed that the triple $(H \supset X ; \Gamma)$ satisfies the partial KKM principle.

(5) For Horvath's convex space $(X ; \Gamma)$ with the weak Van de Vel property is a KKM space, where $\Gamma_{A}:=[[A]]$ for each $A \in\langle X\rangle$.

(6) A $\mathbb{B}$-space due to Briec and Horvath is a KKM space.

(7) Recently, Kulpa and Szymanski [45] found some partial KKM spaces which are not KKM spaces.

Now we have the following diagram for triples $(E, D ; \Gamma)$ :

$$
\begin{aligned}
\text { Simplex } & \Longrightarrow \text { Convex subset of a t.v.s. } \\
& \Longrightarrow \text { Lassonde type convex space } \\
& \Longrightarrow \text { H-space } \\
& \Longrightarrow \text { G-convex space } \\
& \Longrightarrow \phi_{A} \text {-space } \\
& \Longrightarrow \text { KKM space } \\
& \Longrightarrow \text { Partial KKM space } \\
& \Longrightarrow \text { Abstract convex space. }
\end{aligned}
$$

The following whole intersection property for the mapvalues of a KKM map is a standard form of the KKM type theorems $[20,23,24]$.
Theorem A. Let $(E, D ; \Gamma)$ be an abstract convex space, the identity map $1_{E} \in \mathfrak{K} \mathfrak{S}(E, D, E)\left(\right.$ resp., $\left.1_{E} \in \mathfrak{K} \mathfrak{D}(E, D, E)\right)$, and $G: D \multimap E$ be a multimap satisfying the following:

(1) G has closed (resp., open) values,

(2) $\Gamma_{N} \subset G(N)$ for any $N \in\langle D\rangle$ (i.e., $G$ is a KKM map).

Then $\{G(z)\}_{z \in D}$ has the finite intersection property.

Further, if

(3) $\bigcap_{z \in M} \overline{G(z)}$ is compact for some $M \in\langle D\rangle$,

then one has

$$
\bigcap_{y \in D} \overline{G(y)} \neq \emptyset
$$

Since all of the spaces in Section 3 are $\phi_{A}$-spaces and hence KKM spaces, Theorem A can be applied to them, that is, Theorem 1-11 for the case (3).

Consider the following related four conditions for a map $G: D \multimap E$.
(a) $\bigcap_{z \in D} \overline{G(z)} \neq \emptyset$ implies $\bigcap_{z \in D} G(z) \neq \emptyset$.
(b) $\bigcap_{z \in D} \overline{G(z)}=\overline{\bigcap_{z \in D} G(z)}$ ( $G$ is intersectionally closed valued).
(c) $\bigcap_{z \in D} \overline{G(z)}=\bigcap_{z \in D} G(z)$ (G is transfer closed-valued).
(d) $G$ is closed valued.

From the partial KKM principle we have a whole intersection property of the Fan type. The following is given in $[20,23,24]$.

Theorem B. Let $(E, D ; \Gamma)$ be a partial KKM space (i.e., $1_{E} \in$ $\mathfrak{i} \mathfrak{C}(E, D, E))$ and $G: D \multimap E$ a map such that

(1) $\bar{G}$ is a KKM map (i.e., $\Gamma_{A} \subset \bar{G}(A)$ for all $A \in\langle D\rangle$ ),

(2) there exists a nonempty compact subset $K$ of $E$ such that either

(i) $\bigcap_{z \in M} \overline{G(z)} \subset K$ for some $M \in\langle D\rangle$,

(ii) or for each $N \in\langle D\rangle$, there exists a compact $\Gamma$ convex subset $L_{N}$ of $E$ relative to some $D^{\prime} \subset D$ such that $N \subset D^{\prime}$ and

$$
\overline{L_{N}} \cap \bigcap_{z \in D^{\prime}} \overline{G(z)} \subset K \text {. }
$$

Then one has $K \cap \bigcap_{z \in D} \overline{G(z)} \neq \emptyset$.

Furthermore,

$(\alpha)$ if $G$ is transfer closed valued, then $K \cap \bigcap\{G(z): z \in$ $D\} \neq \emptyset$;

( $\beta$ ) if $G$ is intersectionally closed valued, then $\bigcap\{G(z): z \epsilon$ $D\} \neq \emptyset$.

Remark 16. (1) Every $\phi_{A}$-space is a KKM space. Hence, Theorem B works for $\phi_{A}$-spaces.

(2) We may assume that $K$ is closed. Then, the closure notations in each coercivity condition can be eliminated. 
From the preceding theorem, we obtained several prototypes of minimax inequalities in [29]. Here we give only one of them with its proof for completeness.

Theorem 17 ([29]). Let $(E, D ; \Gamma)$ be a partial KKM space, let $f: D \times E \rightarrow \overline{\mathbb{R}}, g: E \times E \rightarrow \overline{\mathbb{R}}$ and be extended real-valued functions, and let $\alpha, \beta \in \overline{\mathbb{R}}$ such that

(0) for each $x \in E, g(x, x) \leq \beta$,

(1) the map $G: D \multimap E$ defined by $G(z):=\{y \in E$ : $f(z, y) \leq \alpha\}$ for $z \in D$ is intersectionally closed-valued (resp., transfer closed-valued),

(2) for each $y \in E, \operatorname{co}_{\Gamma}\{z \in D: f(z, y)>\alpha\} \subset\{x \in E$ : $g(x, y)>\beta\}$,

(3) the coercivity condition (2) of Theorem B holds.

Then, (a) there exists a $y_{0} \in E$ (resp., $y_{0} \in K$ ) such that $f\left(z, y_{0}\right) \leq \alpha$ for all $z \in D$; and

(b) if $\alpha=\beta:=\sup _{x \in E} g(x, x)$, then one has

$$
\inf _{y \in E} \sup _{z \in D} f(z, y) \leq \sup _{x \in E} g(x, x) .
$$

Lemma 18. Under the hypothesis of Theorem 17, condition (2) implies that $G: D \multimap X$ is a KKM map.

Proof. Suppose, on the contrary, that there exists a finite $N \subset$ $D$ such that $\Gamma_{N} \not \subset G(N)$. Then, there exist a $y \in \Gamma_{N}$ such that $y \notin G(z)$ or $f(z, y)>\alpha$ for all $z \in N$. Hence, $N \subset\{z \in D$ : $f(z, y)>\alpha\}$ and, by (2), we have $\Gamma_{N} \subset\{x \in X: g(x, y)>\beta\}$. Since $y \in \Gamma_{N}$, we have $g(y, y)>\beta$. This contradicts (1).

Proof of Theorem 17. By Lemma, $G$ is a KKM map. Therefore, all the requirements of Theorem B are satisfied. Hence, (a) follows from Theorem B. Note that (b) is a simple consequence of (a).

Remark 19. (1) Comparing Theorem 17 with the original Fan minimax inequality, note that condition (1) generalizes the l.s.c. of $f(x, \cdot),(2)$ generalizes the quasiconcavity of $f(\cdot, y)$ whenever $E=D$ and $f=g$, and (3) generalizes the compactness of the underlying space.

(2) In condition (1), transfer closedness can be replaced by mere closedness. For a long period, instead of closedness, some authors adopted the so-called compactly closedness, transfer compactly closedness, or even by the finitely closedness when $E$ is a convex subset of a t.v.s. Moreover, (1) holds whenever $G$ is l.s.c. This also can be replaced by compactly l.s.c., transfer compactly l.s.c, or finitely l.s.c. This kind of terminology is not essential and generalizes nothing important.

Now we show the following.

Theorem $17 \Rightarrow$ Theorem 11 . Every $\phi_{A}$-space is a partial KKM space. Put $(E, D ; \Gamma):=\left(X, D ; \phi_{A}\right)$ with $\Gamma_{A}:=\phi_{A}\left(\Delta_{|A|-1}\right)$ and $\alpha=\beta:=\gamma$ in Theorem 17 , then it reduces to Theorem 11 .

Remark 20. (1) The above proof shows that Theorem 17 subsumes all of Theorems 1-11 and numerous variants or particular forms of them appeared in previous works.
(2) There are other theorems also containing Theorem 111 ; see $[26,29]$.

\section{Other Types of Generalizations}

This section deals with some of other generalizations of the Fan minimax inequality and vector minimax inequality.

5.1. Generalizations of Quasiconcavity. The compactness, convexity, lower semicontinuity, and quasiconcavity in the inequality are extended or modified by a large number of authors. For example, the quasicocavity is extended to $\gamma$ DQCV by Zhou and Chen in 1988. Further, Lin and Tian in 1993 [31, Theorem 3] defined $\gamma$-DQCV in slightly more general form:

Let $Y$ be a convex subset of a Hausdorff t.v.s. $E$ and let $\emptyset \neq X \subset Y$. A functional $\varphi(x, y): X \times Y \rightarrow \overline{\mathbb{R}}$ is said to be $\gamma$-diagonally quasi-concave $(\gamma$-DQCV) in $x$ if, for any finite subset $\left\{x_{1}, \ldots, x_{m}\right\} \subset X$ and any $x_{\lambda} \in \operatorname{co}\left\{x_{1}, \ldots, x_{m}\right\}$, we have $\min _{1 \leq j \leq m} \varphi\left(x_{j}, x_{\lambda}\right) \leq \gamma$.

Theorem 21 (see [31]). Let $Y$ be a nonempty convex subset of a Hausdorff t.v.s. E, let $\emptyset \neq X \subset Y$, and let $\varphi: X \times Y \rightarrow \overline{\mathbb{R}}$ be a functional such that

(i) $(x, y) \mapsto \varphi(x, y)$ is l.s.c. in $y$,

(ii) $(x, y) \mapsto \varphi(x, y)$ is $\gamma$-DQCV in $x$,

(iii) there exists a nonempty subset $C$ of $X$ such that $\bigcap_{x \in C}\{y \in Y: \varphi(x, y) \leq \gamma\}$ is compact and $C$ is contained in a compact convex subset $B$ of $Y$.

Then there exists a point $y^{*} \in Y$ such that $\varphi\left(x, y^{*}\right) \leq \gamma$ for all $x \in X$.

Lin and Tian [31] proved Theorem 21 by applying the partition of unity argument [this is why Hausdorffness is assumed] and the Brouwer fixed point theorem. Moreover, they showed that Theorem 21 is equivalent to the Fan KKM lemma (where Hausdorffness is redundant). Note that Theorem 21 improves the 1984 inequality of Fan.

Moreover, the $\mathscr{C}$-quasiconcavity due to Hou in 2009 [46] unifies the diagonal transfer quasiconcavity (weaker than quasiconcavity) and the $\mathscr{C}$-concavity (weaker than concavity).

Here we introduce a new usage of $\phi_{A}$-spaces and a new minimax inequality.

Definition 22 (see [46]). Let $X$ be a topological space, and let $D, Y \subset X$. A real function $f: X \times Y \rightarrow \mathbb{R}$ is said to be $\mathscr{C}$-quasiconcave on $D$ if, for any $N=\left\{x^{0}, x^{1}, \ldots, x^{n}\right\} \in\langle D\rangle$, there exists a continuous map $\phi_{N}: \Delta_{n} \rightarrow Y$ such that

$$
\min \left\{f\left(x^{i}, \phi_{N}(\lambda)\right): i \in J\right\} \leq f\left(\phi_{N}(\lambda), \phi_{N}(\lambda)\right)
$$

for all $\lambda:=\left(\lambda_{0}, \lambda_{1}, \ldots, \lambda_{n}\right) \in \Delta_{n}$, where $J:=\left\{i: \lambda_{i} \neq 0\right\}$. Note that $\left(Y, D ;\left\{\phi_{N}\right\}_{N \in\langle D\rangle}\right)$ is a $\phi_{A}$-space. 
In [26], we obtained the following generalization of the Fan minimax inequality:

Theorem 23 (see [26]). Let $X$ be a topological space, $D$ a nonempty subset of $X$, and $f, g: X \times X \rightarrow \mathbb{R}$. Assume that:

(1) $f \leq g$ on $X \times X$,

(2) there exist $x^{1}, \ldots, x^{n} \in D$ such that $K=\bigcap_{i=1}^{n} \overline{G\left(x^{i}\right)}$ is compact where $G(x)=\{z \in X: f(x, z) \leq \mu\}$ and $\mu=\sup _{y \in X} g(y, y)$;

(3) $\left.g\right|_{D \times X}$ is $\mathscr{C}$-quasiconcave on $D$,

(4) for each $x \in D,\{y \in X: f(x, y) \leq \mu\}$ is intersectionally closed [resp., transfer closed].

Then there exists $\widetilde{z} \in X($ resp., $\widetilde{z} \in K)$ such that

$$
\sup _{x \in D} f(x, \tilde{z}) \leq \sup _{y \in X} g(y, y)
$$

holds.

Furthermore, S.-Y. Chang in 2010 extended the $\mathscr{C}$ quasiconcavity to 0-pair-concavity and obtained a new Fan type inequality; see Park [26].

5.2. Vector Ky Fan Minimax Inequality. As in [47, Section 3.2], we obtained a vector Ky Fan minimax inequality in [28].

Definition 24 (see [47]). Let $X$ be a Hausdorff topological space, $Z$ a Hausdorff topological vector space with nonempty, convex, closed, and pointed cone $P$ with Int $P \neq \emptyset$, and $D, Y \subset$ $X$. A function $f: X \times Y \rightarrow Z$ is called $\mathscr{C}$-P-quasiconcave on $A$ if and only if, for any finite subset $\left\{x_{0}, x_{1}, \ldots, x_{n}\right\}$ of $D$, there exists a continuous mapping $\phi_{n}: \Delta_{n} \rightarrow Y$ such that, for any $\lambda=\left(\lambda_{0}, \lambda_{1}, \ldots, \lambda_{n}\right) \in \Delta_{n}$, there exists $i \in J(\lambda)$ such that

$$
f\left(\phi_{n}(\lambda), \phi_{n}(\lambda)\right) \in f\left(x_{i}, \phi_{n}(\lambda)\right)+P,
$$

where $J(\lambda):=\left\{i \in\{0,1, \ldots, n\}: \lambda_{i}>0\right\}$.

In [47], the $\mathscr{C}$-P-quasiconcavity is called the generalized $P$-quasiconcave. In the above definition, note that $\left(Y, D ;\left\{\phi_{n}\right\}\right)$ is a $\phi_{A}$-space.

Definition 25 (see [47]). Let $X$ be a Hausdorff topological space, and let $Z$ be a Hausdorff topological vector space with nonempty, convex, closed, and pointed cone $P$ with Int $P \neq \emptyset$. A vector-valued function $f: X \rightarrow Z$ is called $P$-continuous at $x_{0} \in X$ if and only if, for any open neighborhood $V$ of the zero element in $Z$, there exists an open neighborhood $U$ of $x_{0}$ in $X$ such that, for any $x \in U, f(x) \in f\left(x_{0}\right)+V+P$. In particular, $f$ is called $P$-continuous on $X$ if and only if $f$ is $P$-continuous at every point of $X$.

Theorem 26 (see [28]). Let $X$ be a nonempty and compact subset of a Hausdorff topological vector space $E$, and let $Z$ be a Hausdorff topological vector space with nonempty, convex, closed, and pointed cone $P$ with Int $P \neq \emptyset$. A mapping $f: X \times$ $X \rightarrow Z$ satisfies the following: (i) for any fixed $y \in X, x \mapsto f(x, y)$ is P-continuous;

(ii) for any fixed $x \in X, y \mapsto f(x, y)$ is $\mathscr{C}$-P-quasiconcave on $X$;

(iii) for any $x \in X, f(x, x) \notin \operatorname{Int} P$.

Then there exists $x^{*} \in X$ such that $f\left(x^{*}, y\right) \notin \operatorname{Int} P$ for any $y \in X$.

\section{Comments and Historical Remarks}

Recall that the main applications of recent generalized KKM theorems are as follows; see $[11,30]$ :

Vector variational-type inequalities,

Various quasiequilibrium problems,

Eigenvector problems,

Set-valued minimax inequality,

Fixed point theorems,

Generalizations of Nash equilibrium theorem,

Variational inclusion problem,

Simultaneous nonlinear inequalities problem,

Iferential inclusion problem,

(Vector mixed) quasivariational inequality,

(Vector mixed) quasicomplementarity problem,

Traffic network problem,

Quasi-monotone vector equilibrium problem,

Generalized vector equilibrium problem,

Generalized (implicit) vector variational-like inequality,

Set equilibrium problem,

Set-valued mixed (quasi)variational inequalities,

Variational-hemivariational inequalities.

In 2006-2009, we proposed new concepts of abstract convex spaces and the (partial) KKM spaces which are proper generalizations of G-convex spaces and adequate to establish the KKM theory; see $[3,18,19]$ and the references therein. The partial KKM principle for an abstract convex space is an abstract form of the classical KKM theorem. A partial KKM space is an abstract convex space satisfying the partial KKM principle. A KKM space is an abstract convex space satisfying the partial KKM principle and its "open" version. Now the KKM theory becomes the study of spaces satisfying the partial KKM principle.

In our work [3], we clearly derived a sequence of a dozen statements which characterize the KKM spaces and equivalent formulations of the partial KKM principle. We add more than a dozen statements as their applications, including generalized formulations of von Neumann minimax theorem, von Neumann intersection lemma, the Nash equilibrium theorem, and the Fan type minimax inequalities for any KKM spaces. Consequently, [3] unifies and enlarges previously known several proper examples of such statements for particular types of KKM spaces. 


\section{References}

[1] B. Knaster, K. Kuratowski, and S. Mazurkiewicz, "Ein beweis des fixpunktsatzes für $n$ simplexe," Fundamenta Mathematicae, vol. 14, pp. 132-137, 1929.

[2] S. Park, "Ninety years of the Brouwer fixed point theorem," Vietnam Journal of Mathematics, vol. 27, no. 3, pp. 187-222, 1999.

[3] S. Park, "The KKM principle in abstract convex spaces: equivalent formulations and applications," Nonlinear Analysis, vol. 73, no. 4, pp. 1028-1042, 2010.

[4] K. Fan, "A generalization of Tychonoff's fixed point theorem," Mathematische Annalen, vol. 142, pp. 305-310, 1961.

[5] K. Fan, "A minimax inequality and applications," in Inequalities III, pp. 103-113, Academic Press, New York, NY, USA, 1972.

[6] K. Fan, "Sur un théorème minimax," Comptes Rendus de l'Académie des Sciences, vol. 259, pp. 3925-3928, 1964.

[7] K. Fan, "Applications of a theorem concerning sets with convex sections," Mathematische Annalen, vol. 163, pp. 189-203, 1966.

[8] K. Fan, "Extensions of two fixed point theorems of F. E. Browder," Mathematische Zeitschrift, vol. 112, pp. 234-240, 1969.

[9] K. Fan, "Fixed-point and related theorems for noncompact convex sets," in Game Theory and Related Topics, O. Moeshlin and D. Pallaschke, Eds., pp. 151-156, North-Holland, Amsterdam, The Netherlands, 1979.

[10] K. Fan, "Some properties of convex sets related to fixed point theorems," Mathematische Annalen, vol. 266, no. 4, pp. 519-537, 1984.

[11] S. Park, "Recent applications of the Fan-KKM theorem," in Proceedings of the RIMS International Workshop on Nonlinear Analysis and Convex Analysis, RIMS Kôkyûroku, Kyoto University, Kyoto, Japan, August 2012.

[12] M. Lassonde, "On the use of KKM multifunctions in fixed point theory and related topics," Journal of Mathematical Analysis and Applications, vol. 97, no. 1, pp. 151-201, 1983.

[13] C. D. Horvath, "Some results on multivalued mappings and inequalities without convexity," in Nonlinear and Convex Analysis: Proceedings in Honor of Ky Fan, B. L. Lin and S. Simons, Eds., pp. 99-106, Marcel Dekker, New York, NY, USA, 1987.

[14] C. D. Horvath, "Convexité généralisée et application," Séminaire de Mathématiques Supérieures, vol. 110, pp. 81-99, 1990.

[15] C. D. Horvath, "Contractibility and generalized convexity," Journal of Mathematical Analysis and Applications, vol. 156, no. 2, pp. 341-357, 1991.

[16] C. D. Horvath, "Extension and selection theorems in topological spaces with a generalized convexity structure," Toulouse, vol. 2, no. 2, pp. 253-269, 1993.

[17] S. Park, "Various subclasses of abstract convex spaces for the KKM theory," Proceedings of the National Institute of Mathematical Science, vol. 2, no. 2, pp. 35-47, 2007.

[18] S. Park, "Elements of the KKM theory on abstract convex spaces," Journal of the Korean Mathematical Society, vol. 45, no. 1, pp. 1-27, 2008.

[19] S. Park, "New foundations of the KKM theory," Journal of Nonlinear and Convex Analysis, vol. 9, no. 3, pp. 331-350, 2008.

[20] S. Park, "General KKM theorems for abstract convex spaces," Journal of Informatics and Mathematical Sciences, vol. 1, no. 1, pp. 1-13, 2009.

[21] S. Park, "A brief history of the KKM theory," RIMS Kôkyûroku, vol. 1643, pp. 1-16, 2009.

[22] S. Park, "Remarks on some basic concepts in the KKM theory," Nonlinear Analysis, vol. 74, no. 7, pp. 2439-2447, 2011.
[23] S. Park, "A genesis of general KKM theorems for abstract convex spaces," Journal of Nonlinear Analysis and Optimization, vol. 2, no. 1, pp. 133-146, 2011.

[24] S. Park, "Remarks on certain coercivity in general KKM theorems," Nonlinear Analysis Forum, vol. 16, pp. 1-10, 2011.

[25] S. Park, "Abstract convex spaces, KKM spaces, and $\phi_{A}$-spaces," Nonlinear Analysis Forum, vol. 17, pp. 1-10, 2012.

[26] S. Park, "On S.-Y. Chang's inequalities and Nash equilibria," Journal of Nonlinear and Convex Analysis, vol. 12, pp. 455-471, 2010.

[27] S. Park, "The Fan minimax inequality implies the Nash equilibrium theorem," Applied Mathematics Letters, vol. 24, no. 12, pp. 2206-2210, 2011.

[28] S. Park, "Remarks on a generalized KKM theorem without convex hull," Communications on Pure and Applied Analysis, vol. 19, no. 4, pp. 57-65, 2012.

[29] S. Park, "On some new Ky Fan type minimax inequalities in abstract convex spaces," in Proceedings of the 7th International Conference on Nonlinear Analysis and Convex Analysis (NACA '11), August 2011.

[30] S. Park, "Recent applications of the generalized KKM theorems," in Proceedings of the Asian Conference on Nonlinear Analysis and Optimization, Yokohama Publishers, Shimane, Japan, 2012.

[31] Y. J. Lin and G. Q. Tian, "Minimax inequalities equivalent to the Fan-Knaster-Kuratowski-Mazurkiewicz theorems," Applied Mathematics and Optimization, vol. 28, no. 2, pp. 173-179, 1993.

[32] G. Allen, "Variational inequalities, complementarity problems, and duality theorems," Journal of Mathematical Analysis and Applications, vol. 58, no. 1, pp. 1-10, 1977.

[33] J. F. Nash, Jr., "Equilibrium points in n-person games," Proceedings of the National Academy of Sciences of the United States of America, vol. 36, pp. 48-49, 1950.

[34] J. Nash, "Non-cooperative games," Annals of Mathematics, vol. 54, pp. 286-295, 1951.

[35] S. Park, "Foundations of the KKM theory via coincidences of composites of upper semicontinuous maps," Journal of the Korean Mathematical Society, vol. 31, no. 3, pp. 493-519, 1994.

[36] S. Park, "Remarks on topologies of generalized convex spaces," Nonlinear Functional Analysis and Applications, vol. 5, no. 2, pp. 67-79, 2000.

[37] S. Y. Chang, "A generalization of KKM principle and its applications," Soochow Journal of Mathematics, vol. 15, no. 1, pp. 7-17, 1989.

[38] S. Park, "Remarks on some variational inequalities," Bulletin of the Korean Mathematical Society, vol. 28, no. 2, pp. 163-174, 1991.

[39] S. Park, "On minimax inequalities on spaces having certain contractible subsets," Bulletin of the Australian Mathematical Society, vol. 47, no. 1, pp. 25-40, 1993.

[40] C. Bardaro and R. Ceppitelli, "Some further generalizations of Knaster-Kuratowski-Mazurkiewicz theorem and minimax inequalities," Journal of Mathematical Analysis and Applications, vol. 132, no. 2, pp. 484-490, 1988.

[41] S. Park and H. Kim, "Coincidence theorems for admissible multifunctions on generalized convex spaces," Journal of Mathematical Analysis and Applications, vol. 197, no. 1, pp. 173-187, 1996.

[42] S. Park and H. Kim, "Foundations of the KKM theory on generalized convex spaces," Journal of Mathematical Analysis and Applications, vol. 209, no. 2, pp. 551-571, 1997. 
[43] S. Park, "Comments on the KKM theory on $\varphi_{A}$-spaces," Panamerican Mathematical Journal, vol. 18, no. 2, pp. 61-71, 2008.

[44] S. Park, "Generalized convex spaces, $L$-spaces, and FC-spaces," Journal of Global Optimization, vol. 45, no. 2, pp. 203-210, 2009.

[45] W. Kulpa and A. Szymanski, "Some remarks on Park's abstract convex spaces," Preprint.

[46] J.-C. Hou, "Characterization of the existence of a pure-strategy Nash equilibrium," Applied Mathematics Letters, vol. 22, no. 5, pp. 689-692, 2009.

[47] Z. Yang and Y. J. Pu, "Generalized Knaster-Kuratowski-Mazurkiewicz theorem without convex hull," Journal of Optimization Theory and Applications, vol. 154, no. 1, pp. 17-29, 2012. 


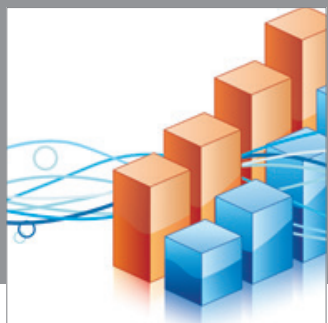

Advances in

Operations Research

mansans

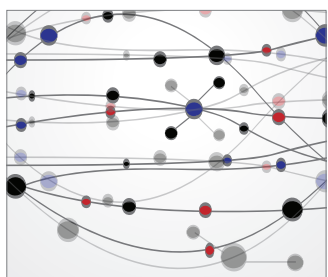

The Scientific World Journal
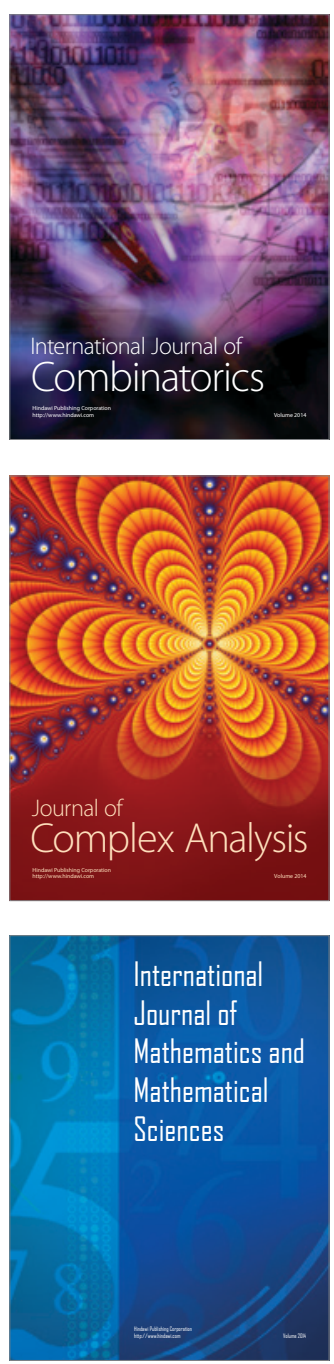
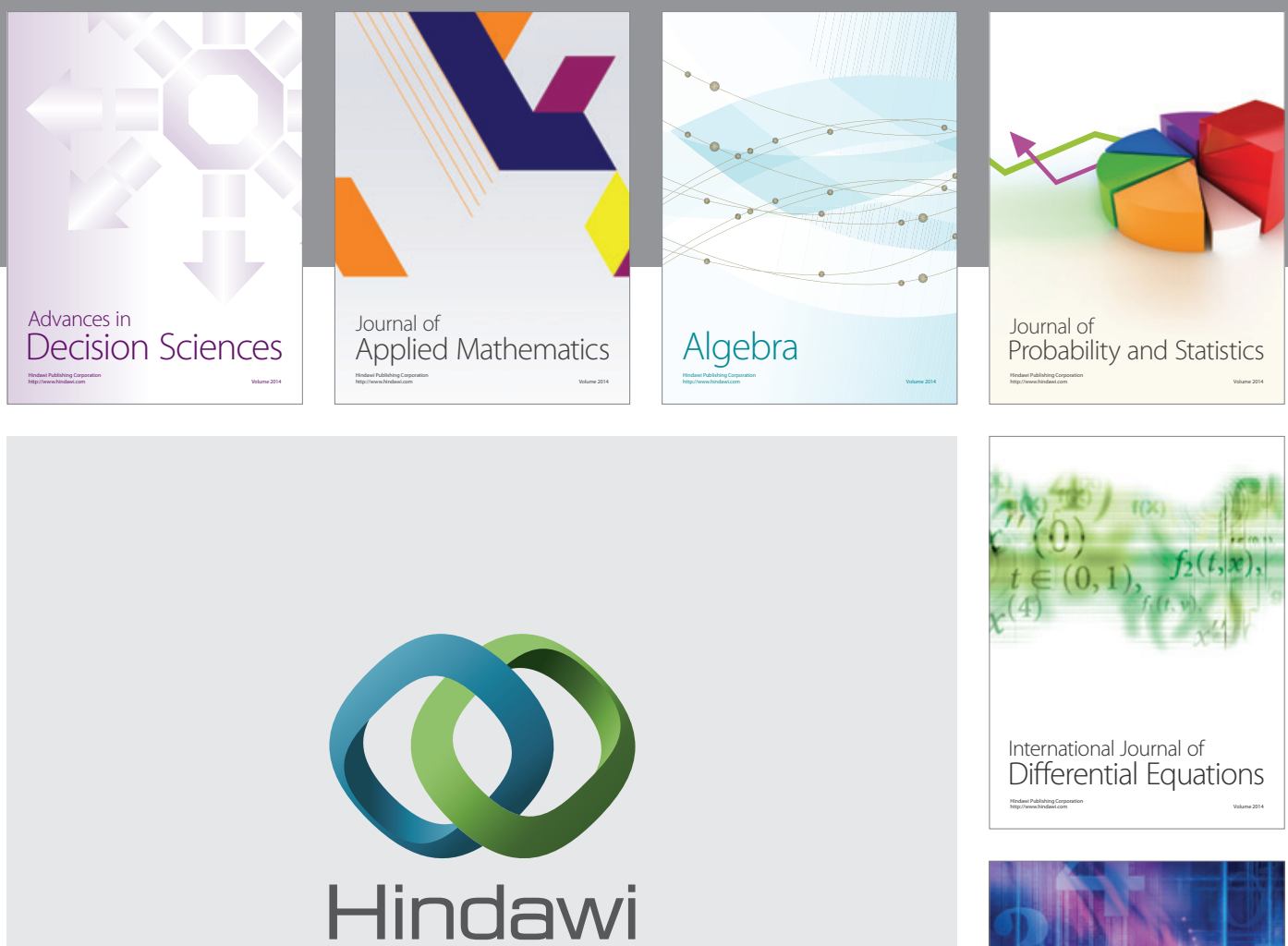

Submit your manuscripts at http://www.hindawi.com
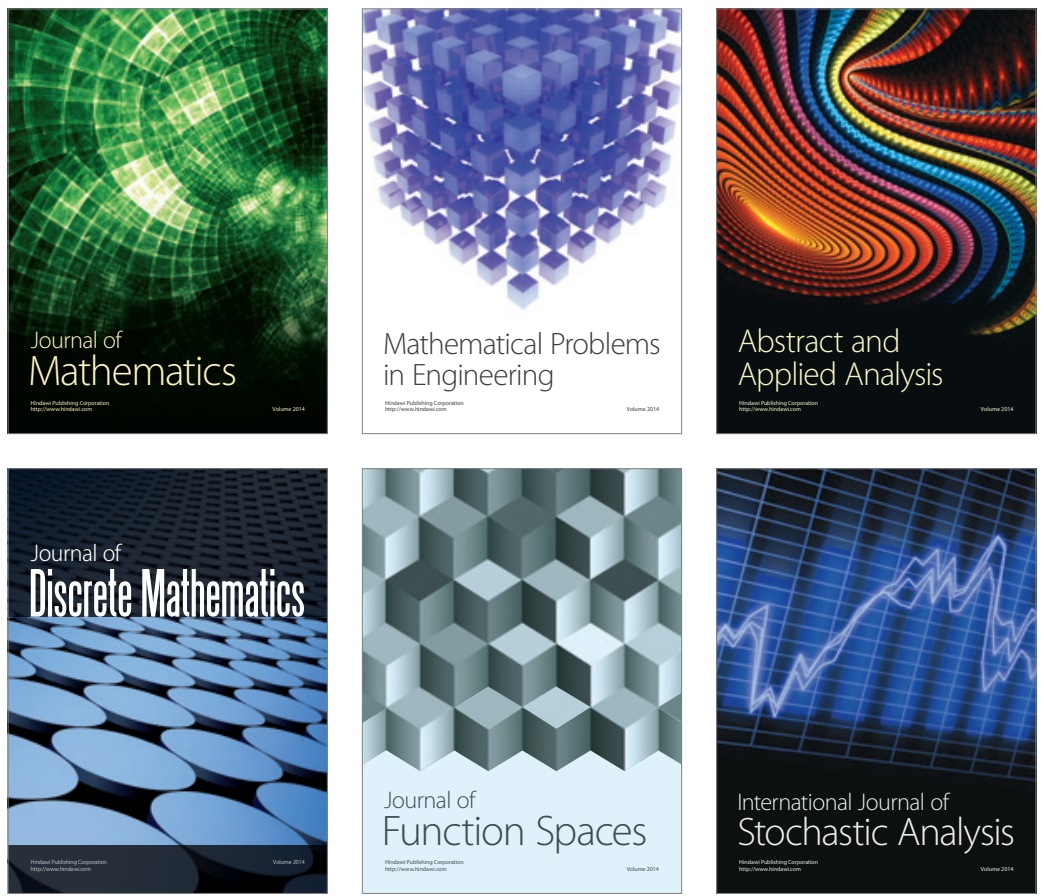

Journal of

Function Spaces

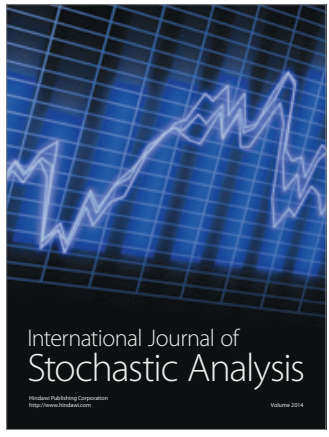

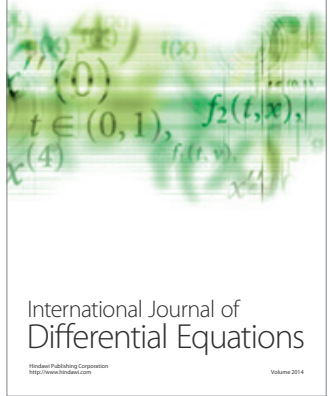
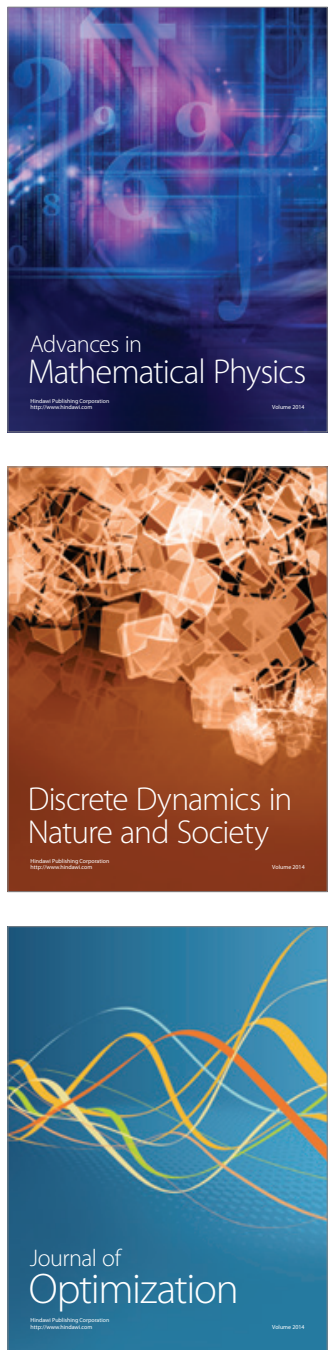